\title{
Correction: The topoisomerase I- and p53-binding protein topors is differentially expressed in normal and malignant human tissues and may function as a tumor suppressor
}

\author{
Ahamed Saleem ${ }^{1} \cdot$ Jayeeta Dutta ${ }^{1} \cdot$ Diptee Malegaonkar $^{1} \cdot$ Farheena Rasheed $^{1} \cdot$ Zeshaan Rasheed $^{1} \cdot$

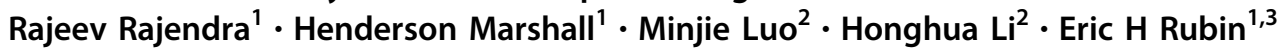

Published online: 11 July 2019

(c) Springer Nature Limited 2019

\section{Correction to: Oncogene}

https://doi.org/10.1038/sj.onc.1207700
Following publication of this article the author Minjie Luo noted that their name had been published incorrectly as Minje Luo.

The original article can be found online at https://doi.org/10.1038/sj. onc. 1207700 .

Eric H Rubin

ehrubin@umdnj.edu

1 Department of Pharmacology, the Cancer Institute of New Jersey, Robert Wood Johnson Medical School, University of Medicine and Dentistry of New Jersey, New Brunswick, NJ, USA

2 Department of Molecular Genetics and Microbiology, the Cancer Institute of New Jersey, Robert Wood Johnson Medical School, University of Medicine and Dentistry of New Jersey,

New Brunswick, NJ, USA

3 Department of Medicine, the Cancer Institute of New Jersey, Robert Wood Johnson Medical School, University of Medicine and Dentistry of New Jersey, New Brunswick, NJ, USA 